Relations among western corn rootworm resistance traits and elements concentration in maize germplasm roots

Odnosi između svojstava otpornosti na kukuruznu zlaticu i koncentracije elemenata u korijenu u germplazmi kukuruza

Brkić, A., Brkić, I., Raspudić, E., Brmež, M., Brkić, J., Šimić, D.

Poljoprivreda/Agriculture

ISSN: 1848-8080 (Online)

ISSN: 1330-7142 (Print)

http://dx.doi.org/10.18047/poljo.21.1.01

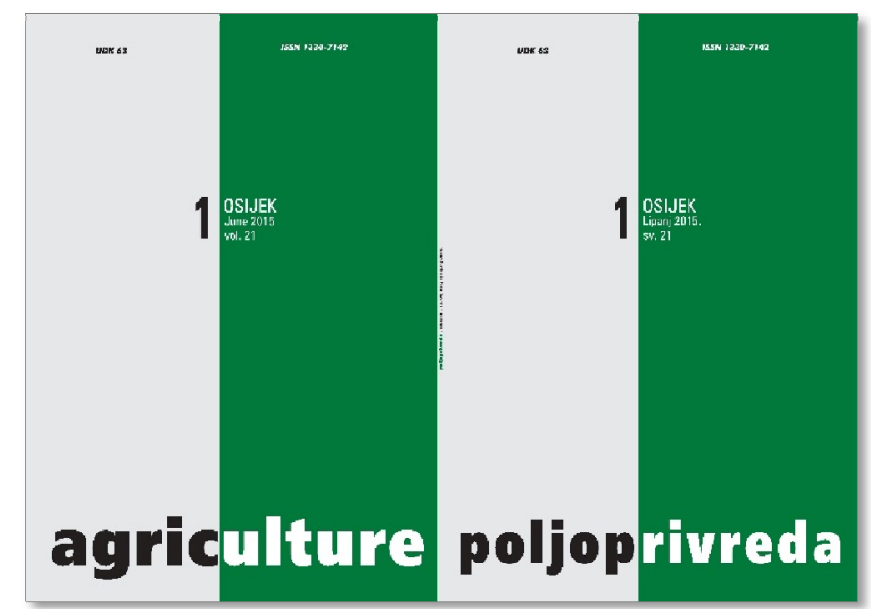

Poljoprivredni fakultet u Osijeku, Poljoprivredni institut Osijek Faculty of Agriculture in Osijek, Agricultural Institute Osijek 
ISSN 1330-7142

UDK: 632.768

DOI: $10.18047 /$ poljo.21.1.01

\section{RELATIONS AMONG WESTERN CORN ROOTWORM RESISTANCE TRAITS AND ELEMENTS CONCENTRATION IN MAIZE GERMPLASM ROOTS}

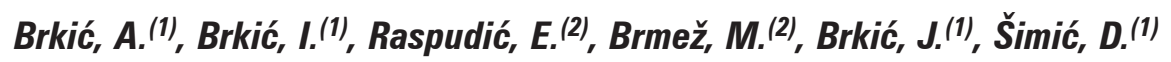

Original scientific paper

Izvorni znanstveni članak

SUMMARY

Western corn rootworm - WCR (Diabrotica virgifera virgifera LeConte) is an important maize pest in Croatia. Using native resistance of maize germplasm could reduce chemical treatments and other costs in maize production. Objectives of this study were: i) to assess variability of WCR resistance traits (root injury, regrowth and size) and concentrations of nine elements in roots of 128 maize genotypes, and ii) to determine correlations among the traits and ion concentrations. Results revealed high variability of maize genotypes for both WCR resistance traits and ion concentrations. Significant moderate negative correlations (>-0.4) were detected between root injury and boron as well as between root regrowth and iron, manganese and zinc concentrations in root. Consequently, ion concentration in maize roots might have an impact on WCR resistance research.

Key-words: western corn rootworm, root injury, ion concentration, maize germplasm

\section{INTRODUCTION}

Western corn rootworm - WCR (Diabrotica virgifera virgifera LeConte) is a major pest in USA and from early 1990s the pest spread substantially in Europe (Gray et al., 2009). Very soon after its discovery in 1995, WCR became one of the most important maize pests in Croatia. Significant efforts in research on native resistance of maize against WCR has been carried out in eastern Croatia (Ivezić et al., 2006, 2011; Šimić et al., 2007) for the purpose of identification of native resistance germplasm sources. Native resistant maize hybrids would reduce costs for growers, and represent a sustainable option in maize production.

The functional genomics of all ions (elements) called ionomics (Lahner et al., 2003), has been established recently, enabling measurement of concentrations of many elements simultaneously, which requires the application of high-throughput elemental analysis technologies. Accordingly, the root ionome of a maize plant represents its mineral and trace element content, and it is dependent on many factors including soil properties, several physiological processes and biotic environment including WCR. Furthermore, Boyd (2007) demonstrated that plant can be defended against some natural enemies by accumulating some elements sug- gesting that defensive effects of elements in plants are more widespread than previously believed. To date, several studies characterized ionome of maize shoot, leaves and grain (e.g. Sorić et al., 2011; Šimić et al., 2012; Baxter et al., 2013; Mascher et al., 2014) but none dealt with maize root ionome in the context of WCR native resistance.

Objectives of this study were to assess variability of WCR resistance traits (root injury - Rl, root regrowth $R R$, and root size $-R S$ ), concentrations of nine elements in roots of 128 maize genotypes, and to determine correlations among the traits and ion concentrations.

\section{MATERIAL AND METHODS}

Field trial was set out using rectangular $(16 \times 8)$ lattice incomplete block design with 128 genotypes planted in 2009 at one location (Osijek) in two replications. Genotype set consisted of 120 inbred lines from different heterotic groups developed at Agricultural Institute 0sijek and 8 synthetic populations from USA (courtesy of Bruce

(1) Ph.D. Andrija Brkić (andrija.brkic@poljinos.hr), Ph.D. Ivan Brkić, Ph.D. Josip Brkić, Ph.D. Domagoj Šimić - Agricultural Institute Osijek, Južno predgrađe 17, Osijek 31000, Croatia, (2) Prof. Dr. Emilija Raspudić, Prof. Dr. Mirjana Brmež - Josip Juraj Strossmayer University of Osijek, Faculty of Agriculture in Osijek, Kralja Petra Svačića 1d, 31000 Osijek, Croatia 
Hibbard, ARS-USDA, University of Missouri). Genetic background of the genotypes was previously given by Brkić (2012). US populations already showed certain levels of resistance in US conditions (Hibbard et al., 2007; Prischmann et al., 2007).The research was carried out under natural WCR infestation conditions. Soil was eutric cambisol, the soil type of moderate fertility with no metal imbalances. Chemical properties of the soil corresponded to data presented by Simić et al. (2009). Fertilizers were given according to usual requirements for high yielding maize, taking into account the soil characteristics and the previous cropping. No additional fertilizers with micronutrients were applied.

In early July plants (roots) were manually pulled out of the soil, tagged, transported to water station and washed clean. After washing three main root traits were estimated - root injury with 0-3 lowa State University node injury scale (Oleson et al., 2005), and root regrowth and size with 1-6 Eiben reversed scale (Rogers et al., 1975). Ion concentrations of nine elements in roots (boron - $\mathrm{B}$, cadmium - $\mathrm{Cd}$, copper - $\mathrm{Cu}$, iron - $\mathrm{Fe}$, magnesium - Mg, manganese - Mn, phosphorus - $P_{\text {, }}$ strontium - $\mathrm{Sr}$, zinc - $\mathrm{Zn}$ ) were determined by inductively coupled plasma - optical emission spectrometry
(ICP-OES) technique (Optima 2100 DV by PerkinElmer). Microwave digestion of root samples $\left(\mathrm{HNO}_{3}\right.$ and $\mathrm{H}_{2} \mathrm{O}_{2}$ added) was performed with Mars Extraction microwave oven in the laboratory of the Research Institute for Soil Science and Agricultural Chemistry of Hungarian Academy of Science in Budapest, Hungary (Zarcinas et al., 1987; Sorić et al., 2011). Ion concentrations were expressed on root dry matter basis.

Analysis of variance, coefficients of variation, repeatability estimates for lattice designs and correlation coefficients were calculated using PLABSTAT program package (Utz, 1995).

\section{RESULTS AND DISCUSSION}

Analysis of variance showed that all sources of variation were highly significant $(\mathrm{P}<0.01)$ for all three WCR resistant traits, except effect of replication on root injury, which was not significant (Table 1). Previous study by Brkić (2012) showed the same variability estimates, where only the effect of replication for root injury was not significant. Sources of variation for root ion concentrations were also highly significant, except effect of replication for $\mathrm{Mg}$ concentration, which was significant $(\mathrm{P}<0.05$, Table 2$)$.

Table 1. ANOVA for WCR resistance traits

Tablica 1. Analiza varijance za svojstva otpornosti na kukuruznu zlaticu

\begin{tabular}{|c|c|c|c|c|}
\hline \multirow{2}{*}{$\begin{array}{l}\text { Source of variation } \\
\text { Izvor variranja }\end{array}$} & \multirow{2}{*}{$\begin{array}{l}\text { Df } \\
\text { Ss }\end{array}$} & \multicolumn{3}{|c|}{ F-value / F-vrijednost } \\
\hline & & $\begin{array}{c}\text { Root injury (RI) } \\
\text { Oštećenje korijena }\end{array}$ & $\begin{array}{c}\text { Root regrowth (RR) } \\
\text { Sekundarni porast korijena }\end{array}$ & $\begin{array}{l}\text { Root size (RI) } \\
\text { Veličina korijena }\end{array}$ \\
\hline $\begin{array}{l}\text { Replication } \\
\text { Ponavljanje }\end{array}$ & 1 & 0.07 & $85.63^{* *}$ & $39.47^{* *}$ \\
\hline $\begin{array}{l}\text { Genotype } \\
\text { Genotip }\end{array}$ & 127 & $7.24^{* *}$ & $2.56^{* *}$ & $2.72^{* *}$ \\
\hline $\begin{array}{l}\text { Block } \\
\text { Blok }\end{array}$ & 14 & $35.49 * *$ & $5.07^{* *}$ & $5.45^{* *}$ \\
\hline
\end{tabular}

** , ${ }^{*}$ Significant at $0.01,0.05$ probability level, respectively - Statistički značajno na razini vjerojatnosti 0,01, odnosno 0,05

Table 2. ANOVA for root ion concentrations

Tablica 2. Analiza varijance za koncentracije iona u korijenu

\begin{tabular}{|c|c|c|c|c|c|}
\hline \multirow{2}{*}{$\begin{array}{l}\text { Source of variation } \\
\text { Izvor variranja }\end{array}$} & \multicolumn{5}{|c|}{ F-value / F-vrijednost } \\
\hline & B & Cd & Cu & $\mathrm{Fe}$ & K \\
\hline Replication / Ponavljanje & $14.30^{* *}$ & $130.23^{* *}$ & $38.13^{* *}$ & $15.79 * *$ & $8.66^{* *}$ \\
\hline Genotype / Genotip & $3.29 * *$ & $4.27^{* *}$ & $2.29 * *$ & $2.31 * *$ & $3.90^{* *}$ \\
\hline Block / Blok & $3.85^{* *}$ & $2.69 * *$ & $5.33^{* *}$ & $5.12^{* *}$ & $4.19^{* *}$ \\
\hline \multirow{2}{*}{$\begin{array}{l}\text { Source of variation } \\
\text { Izvor variranja }\end{array}$} & \multicolumn{5}{|c|}{ F-value / F-vrijednost } \\
\hline & $\mathbf{M g}$ & Mn & $\mathbf{P}$ & Sr & $\mathrm{Zn}$ \\
\hline Replication / Ponavljanje & $4.18^{*}$ & $9.14^{* *}$ & $28.12^{* *}$ & $90.41^{* *}$ & $43.56^{* *}$ \\
\hline Genotype / Genotip & $2.43^{* *}$ & $2.21 * *$ & $5.89 * *$ & $2.36^{* *}$ & $3.83^{* *}$ \\
\hline Block / Blok & $3.27^{* *}$ & $4.50^{* *}$ & $5.65^{* *}$ & $2.49 * *$ & $16.06^{* *}$ \\
\hline
\end{tabular}

**, ${ }^{*}$ Significant at $0.01,0.05$ probability level, respectively - Statistički značajno na razini vjerojatnosti 0,01, odnosno 0,05 
Among all traits, iron, manganese and boron concentration had the highest coefficients of variation $(\mathrm{Fe}=56.0 \%, \mathrm{Mn}=53.5 \%, \mathrm{~B}=47.6 \%)$ (Table 3). Higher repeatabilities of root injury $(75.1 \%)$, phosphorus concentration $(70.5 \%)$ and zinc concentration $(70.4 \%)$ indicate that these traits can be reliably identified in maize genotypes (Table 3). In a comparable study by Šimić et al. (2007) repeatability estimates for root injury were lower $(16.6 \%)$, while estimates for root regrowth were much higher $(89.3 \%)$, under both artificial and natural infestation treatments.

Table 3. Mean, coefficient of variation and repeatability of WCR resistance traits and root ion concentrations Tablica 3. Srednje vrijednosti, koeficijent varijacije i ponovljivost za svojstva otpornosti na kukuruznu zlaticu i koncentracije iona u korijenu

\begin{tabular}{|l|c|c|c|}
\hline Trait / Svojstvo & $\begin{array}{c}\text { Mean } \pm \text { SE } \\
\text { Srednja vrijednost i standardna pogreška }\end{array}$ & $\begin{array}{c}\text { C.V. (\%) } \\
\text { Koeficijent varijacije (\%) }\end{array}$ & $\begin{array}{c}\text { Repeatability (\%) } \\
\text { Ponovljivost (\%) }\end{array}$ \\
\hline Injury / Oštećenje & $1.44 \pm 0.14$ & 13.8 & 75.1 \\
\hline Regrowth / Sekundarni porast & $3.97 \pm 0.46$ & 16.5 & 43.3 \\
\hline Size / Veličina & $3.63 \pm 0.39$ & 15.0 & 45.8 \\
\hline B $(\mathrm{mg} / \mathrm{kg})$ & $4.80 \pm 1.62$ & 47.6 & 53.0 \\
\hline Cd $(\mathrm{mg} / \mathrm{kg})$ & $0.25 \pm 0.04$ & 20.2 & 61.9 \\
\hline Cu $(\mathrm{mg} / \mathrm{kg})$ & $10.99 \pm 1.73$ & 22.3 & 38.7 \\
\hline Fe $(\mathrm{mg} / \mathrm{kg})$ & $3518.24 \pm 1393.83$ & 56.0 & 39.1 \\
\hline $\mathbf{M g}(\mathrm{mg} / \mathrm{kg})$ & $1581.59 \pm 221.90$ & 19.8 & 41.5 \\
\hline $\mathbf{M n}(\mathrm{mg} / \mathrm{kg})$ & $93.47 \pm 35.38$ & 53.5 & 37.4 \\
\hline $\mathbf{P}(\mathrm{mg} / \mathrm{kg})$ & $1760.15 \pm 107.93$ & 8.7 & 70.5 \\
\hline $\mathbf{S r}(\mathrm{mg} / \mathrm{kg})$ & $5.61 \pm 0.63$ & 15.8 & 40.5 \\
\hline $\mathbf{Z n}(\mathrm{mg} / \mathrm{kg})$ & $17.40 \pm 2.23$ & 16.7 & 70.4 \\
\hline
\end{tabular}

Results show significant positive and negative correlations among elements. The highest correlation was between $\mathrm{Mn}$ and $\mathrm{Fe}(0.99)$, as well as between $\mathrm{Mg}$ and $\mathrm{Fe}(0.88), \mathrm{Mn}$ and $\mathrm{Mg}(0.88), \mathrm{Sr}$ and $\mathrm{Mn}(0.80), \mathrm{Sr}$ and $\mathrm{Fe}(0.77), \mathrm{Zn}$ and $\mathrm{Mn}(0.77), \mathrm{Sr}$ and $\mathrm{Mg}(0.76), \mathrm{Zn}$ and $\mathrm{Fe}$ (0.76), $\mathrm{Zn}$ and $\mathrm{Sr}(0.71)$, and $\mathrm{Zn}$ and $\mathrm{Mg}(0.70)$ (Table 4). Significant positive and negative phenotypic correlations between elements in maize ear-leaf were reported by Sorić et al. (2011) (Fe-Cu, Mg-Cu, Mg-Fe, Mg-K, Mn-Fe, $\mathrm{Mn}-\mathrm{Mg}$, Sr-Cu, Sr-K, Sr-Mg and Sr-Mn), and Zdunić et al. (2014) (Fe-Cu, Mn-Fe, Mn-K, Mn-Mg, Sr-Fe, Sr-K, Sr-Mg and Sr-Mn). Another study by Baxter et al. (2013) showed significant correlations of elements in maize grain ( $\mathrm{Fe}-\mathrm{Zn}$, K-P, Mn-P, Mg-P, Cu-K, Mn-Zn, P-Zn, Mg-Zn and others). Neither of these studies included root ionomics.
Significant and positive correlations were determined among WCR resistance traits as well. Highly significant and negative correlations were found out between WCR resistance traits and root mineral concentrations, such as between root injury and B $(-0.41)$, $\mathrm{Cd}(-0.23)$ and $\mathrm{Sr}(-0.26)$, as well as between root regrowth and $\mathrm{Cd}(-0.27), \mathrm{Fe}(-0.46), \mathrm{Mg}(-0.33), \mathrm{Mn}$ $(-0.44), \mathrm{Sr}(-0.36)$ and $\mathrm{Zn}(-0.41)$ (Table 4). Association between WCR root traits and different root ion concentrations suggests that further research should be conducted in order to better understand the correlation between root ionomics and WCR resistance. In this paper, root size did not have any significant correlations with root elemental concentrations.

Table 4. Correlation coefficients of WCR resistance traits and root ion concentrations

Tablica 4. Korelacijski koeficijenti za svojstva otpornosti na kukuruznu zlaticu i koncentracije iona u korijenu

\begin{tabular}{|c|c|c|c|c|c|c|c|c|c|c|c|}
\hline & RI & RR & RS & B & Cd & Cu & Fe & Mg & Mn & P & Sr \\
\hline RR & $0.20^{*}$ & & & & & & & & & & \\
\hline RS & $0.20^{*}$ & $0.58^{* *}$ & & & & & & & & & \\
\hline B & $-0.41^{* *}$ & 0.10 & 0.00 & & & & & & & & \\
\hline Cd & $-0.23^{* *}$ & $-0.27^{* *}$ & -0.02 & 0.13 & & & & & & & \\
\hline $\mathbf{C u}$ & 0.00 & -0.16 & 0.07 & -0.12 & $0.25^{* *}$ & & & & & & \\
\hline $\mathbf{F e}$ & -0.12 & $-0.46^{* *}$ & -0.11 & -0.16 & 0.17 & $0.51^{* *}$ & & & & & \\
\hline $\mathbf{M g}$ & -0.04 & $-0.33^{* *}$ & 0.02 & -0.12 & $0.24^{* *}$ & $0.51^{* *}$ & $0.88^{* *}$ & & & & \\
\hline $\mathbf{M n}$ & -0.13 & $-0.44^{* *}$ & -0.12 & $-0.18^{*}$ & 0.17 & $0.50^{* *}$ & $0.99^{* *}$ & $0.88^{* *}$ & & & \\
\hline $\mathbf{P}$ & $-0.20^{*}$ & -0.01 & -0.15 & $0.19^{*}$ & -0.07 & 0.03 & $-0.26^{* *}$ & $-0.19^{*}$ & $-0.24^{* *}$ & & \\
\hline $\mathbf{S r}$ & $-0.26^{* *}$ & $-0.36^{* *}$ & -0.09 & -0.03 & $0.30^{* *}$ & $0.40^{* *}$ & $0.77^{* *}$ & $0.76^{* *}$ & $0.80^{* *}$ & -0.15 & \\
\hline $\mathbf{Z n}$ & $-0.18^{*}$ & $-0.41^{* *}$ & -0.09 & -0.11 & $0.20^{*}$ & $0.47^{* *}$ & $0.76^{* *}$ & $0.70^{* *}$ & $0.77^{* *}$ & -0.13 & $0.71^{* *}$ \\
\hline
\end{tabular}

**, * Significant correlation at $0.01,0.05$ probability level, respectively - Statistički značajna korelacija na razini vjerojatnosti 0,01, odnosno 0,05; RI = root injury/ oštećenje korijena RR = root regrowth/ sekundarni porast korijena, $\mathrm{RS}=$ root size/ veličina korijena 


\section{CONCLUSION}

Maize genotypes assessed in this research showed high variability for both WCR resistance traits and ion concentration in roots. Also, correlation coefficients of assessed traits showed several significant correlations, particularly correlations between WCR resistance traits (root injury and root regrowth) and some ion concentrations. Therefore, concentration of elements in maize root might have a greater role in WCR resistance research - additional studies are needed in order to better understand physiological mechanisms behind the influence of elemental concentrations on WCR resistance traits.

\section{ACKNOWLEDGEMENTS}

We dedicate this paper to our senior colleagues Prof. Dr. Marija Ivezić and Prof. Dr. Vlado Kovačević for continuous inspiration, support and long-term collaboration.

\section{REFERENCES}

1. Baxter, I.R., Gustin, J.L., Settles, A.M., Hoekenga, O.A. (2013): Ionomic characterization of maize kernels in the intermated B73 $\times$ Mo17 population. Crop Science, 53: 208-220.

doi: 10.2135/cropsci2012.02.0135

2. Boyd, R.S. (2007): The defense hypothesis of elemental hyperaccumulation: status, challenges and new directions. Plant and Soil, 293: 153-176. doi: 10.1007/s11104-007-9240-6

3. Brkić, A. (2012): Genotypic variability of native resistance to western corn rootworm (Diabrotica virgifera virgifera LeConte) in maize germplasm. Ph.D. Thesis, Josip Juraj Strossmayer University of Osijek.

4. Gray, M.E., Sappington, T.W., Miller, N.J., Moeser, J., Bohn, M.O. (2009): Adaptation and invasiveness of western corn rootworm: intensifying research on a worsening pest. Annual Review of Entomology, 54: 303-321. doi: 10.1146/annurev.ento.54.110807.090434

5. Hibbard, B.E., Willmot, D.B., Flint-Garcia, S.A., Darrah, L.L. (2007): Registration of the maize germplasm CRW3(S1)C6 with resistance to western corn rootworm. Journal of Plant Registrations, 1(2): 151-152. doi: $10.3198 / j p r 2006.12 .0774 \mathrm{crg}$

6. Ivezić, M., Tollefson, J.J., Raspudić, E., Brkić, I., Brmež, M., Hibbard, B.E. (2006): Evaluation of corn hybrids for tolerance to corn rootworm (Diabrotica virgifera virgifera LeConte) larval feeding. Cereal Research Communications, 34: 1101-1107.

doi: 10.1556/CRC.34.2006.2-3.244

7. Ivezić, M., Raspudić, E., Majić, I., Tollefson, J.J., Brmež, M., Sarajlić, A., Brkić, A. (2011): Root compensation of seven maize hybrids due to western corn rootworm (Diabrotica virgifera virgifera LeConte) larval injury. Bulgarian Journal of Agricultural Science, 17(1): 107115.

8. Lahner, B., Gong, J., Mahmoudian, M., Smith E.L., Abid, K.B., Rogers, E.E., Guerinot, M.L., Harper, J.F., Ward,
J.M., Mclntyre, L., Schroeder, J.I., Salt, D.E. (2003): Genomic scale profiling of nutrient and trace elements in Arabidopsis thaliana. Nature Biotechnology, 21: 12151221.

doi: $10.1038 / n b t 865$

9. Mascher, M., Gerlach, N., Gahrtz, M., Bucher, M. Scholz, U., Dresselhaus, T. (2014): Sequence and ionomic analysis of divergent strains of maize inbred line B73 with an altered growth phenotype. PLoS ONE, 9(5): http://journals.plos.org/plosone/article?id=10.1371/ journal.pone.0096782 doi:10.1371/journal.pone.0096782.

10. Oleson, J.D., Park, Y.L., Nowatzki, T.M, Tollefson, J.J. (2005): Node-injury scale to evaluate root injury by corn rootworms (Coleoptera: Chrysomelidae). Journal of Economic Entomology, 98: 1-8.

11. Prischmann, D.A., Dashiell, K.E., Schneider, D.J., Hibbard, B.E. (2007): Field screening maize germplasm for resistance and tolerance to western corn rootworm (Col.: Chrysomelidae). Journal of Applied Entomology, 131(6): 406-415. doi: 10.1111/j.1439-0418.2007.01183.x

12. Rogers, R.R., Owens, J.C., Tollefson, J.J., Witkowski, J.F. (1975): Evaluation of commercial corn hybrids for tolerance to corn rootworms. Environmental Entomology, 4: $920-922$.

13. Sorić, R., Ledenčan, T., Zdunić, Z., Jambrović, A., Brkić, I., Lončarić, Z., Kovačević, V., Šimić, D. (2011): Quantitative trait loci for metal accumulation in maize leaf. Maydica, 56: 323-329.

14. Šimić, D., Ivezić, M., Brkić, I., Raspudić, E., Brmež, M., Majić, I., Brkić, A., Ledenčan, T., Tollefson, J.J., Hibbard, B.E. (2007): Environmental and genotypic effects for western corn rootworm tolerance traits in American and European maize trials. Maydica, 52: 425-430.

15. Šimić, D., Zdunić, Z., Jambrović, A., Ledenčan, T., Brkić, I., Duvnjak, V., Kovačević, V. (2009): Relations among six micronutrients in grain determined in a maize population. Poljoprivreda/Agriculture, 15: 15-19.

16. Šimić, D., Mladenović Drinić, S., Zdunić, Z., Jambrović, A., Ledenčan, T., Brkić, J., Brkić, A., Brkić, I. (2012): Quantitative trait loci for biofortification traits in maize grain. J. Hered., 103: 47-54. doi: 10.1093/jhered/esr122

17. Utz, H.F. (1995): PLABSTAT Version M. Ein Computerprogramm zur statistischen Analyse von pflanzenzüchterischen Experimenten. Selbstverlag Universität Hohenheim, Stuttgart.

18. Zarcinas, B.A., Cartwright, B., Spouncer, L.R. (1987): Nitric acid digestion and multi-element analysis of plant material by inductively coupled plasma spectrometry. Communications in Soil Science and Plant Analysis, 18: 131-146. doi: 10.1080/00103628709367806

19. Zdunić, Z., Grljušić, S., Ledenčan, T., Duvnjak, T., Šimić, D. (2014): Quantitative trait loci mapping of metal concentrations in leaves of the maize IBM population. Hereditas, 151: 55-60. doi: $10.1111 / \mathrm{hrd} 2.00048$ 


\section{ODNOSI IZMEĐU SVOJSTAVA OTPORNOSTI NA KUKURUZNU ZLATICU I KONCENTRACIJE ELEMENATA U KORIJENU U GERMPLAZMI KUKURUZA}

\section{SAŽETAK}

Kukuruzna zlatica (Diabrotica virgifera virgifera LeConte) važan je štetnik kukuruza u Hrvatskoj. Korištenje prirodne otpornosti u germplazmi kukuruza moglo bi smanjiti troškove kemijskoga tretiranja, kao i ostale troškove pri proizvodnji kukuruza. Glavni ciljevi ovoga rada bili su: i) procijeniti varijabilnost svojstava otpornosti na kukuruznu zlaticu (oštećenje, sekundarni porast i veličina korijena) i koncentracije devet elemenata u korijenu 128 genotipova kukuruza te ii) utvrditi korelacije između svojstava otpornosti i koncentracije iona. Rezultati su pokazali veliku varijabilnost ispitivanih genotipova i za svojstva otpornosti na kukuruznu zlaticu i za koncentracije iona u korijenu. Značajne negativne korelacije (>-0.4) utvrđene su između oštećenja korijena i bora, kao i između sekundarnoga porasta i željeza, mangana i cinka. Koncentracija iona u korijenu kukuruza mogla bi imati veći utjecaj u istraživanju otpornosti kukuruza na kukuruznu zlaticu.

Ključne riječi: kukuruzna zlatica, oštećenje korijena, koncentracija iona, germplazma kukuruza

(Received on 7 April 2015; accepted on 14 May 2015 - Primljeno 07. travnja 2015.; prihvaćeno 14. svibnja 2015.) 